\title{
Technè
}

La science au service de l'histoire de l'art et de la préservation des biens culturels

$44 \mid 2016$

Archives de l'humanité : les restes humains patrimonialisés

\section{Paléopathologie et épidémiologie sur les collections de musées}

Paleopathology and epidemiology in museum collections

Olivier Dutour et Hélène Coqueugniot

\section{(2) OpenEdition}

12 Journals

\section{Édition électronique}

URL : http://journals.openedition.org/techne/1077

DOI : 10.4000/techne.1077

ISSN : 2534-5168

Éditeur

C2RMF

Édition imprimée

Date de publication : 1 novembre 2016

Pagination : 67-70

ISBN : 978-2-7118-6339-6

ISSN : 1254-7867

Référence électronique

Olivier Dutour et Hélène Coqueugniot, « Paléopathologie et épidémiologie sur les collections de musées », Technè [En ligne], 44 | 2016, mis en ligne le 19 décembre 2019, consulté le 25 juillet 2020. URL : http://journals.openedition.org/techne/1077 ; DOI : https://doi.org/10.4000/techne.1077

\section{(c)}

La revue Technè. La science au service de l'histoire de l'art et de la préservation des biens culturels est mise à disposition selon les termes de la Licence Creative Commons Attribution - Pas d'Utilisation

Commerciale - Pas de Modification 4.0 International. 
Olivier Dutour

Hélène Coqueugniot

\section{Paléopathologie et épidémiologie sur les collections de musées}

Paleopathology and epidemiology in museum collections
Résumé. Les collections ostéologiques humaines ont été constituées à partir de différentes sources (archéo-anthropologiques, anatomiques ou pathologiques). Leur origine détermine différents niveaux d'intérêt paléopathologique et paléoépidémiologique : les collections pathologiques ont un intérêt essentiellement didactique et les collections identifiées, qu'elles soient d'origine anthropologique ou anatomique, permettent une approche méthodologique et paléoépidémiologique.

Mots-clés. Paléopathologie, paléoépidémiologie, collections anthropologiques, collections anatomiques, collections ostéologiques.

\author{
Abstract. The collections of human bones were built up from \\ different sources: archaeo-anthropological, anatomical \\ or pathological. Their origin determines different levels \\ of paleopathological and paleoepidemiological interest: \\ the pathological collections have an essentially didactic interest \\ and the identified collections, whether anthropological \\ or anatomical in origin, enable a methodological \\ and paleoepidemiological approach to be adopted. \\ Keywords. Paleopathology, paleoepidemiology, \\ anthropological collections, anatomical collections, osteological \\ collections.
}

\section{Introduction}

La paléopathologie est une discipline récente, définie en 1913 par un médecin bactériologiste franco-britannique, Sir Marc Armand Ruffer, comme étant « la science des maladies dont on peut démontrer l'existence sur des restes humains et animaux des périodes anciennes ${ }^{1}{ }^{»}$.

Cette science se situe à l'interface de deux grands domaines : celui des sciences biomédicales, dont elle utilise la démarche diagnostique et les méthodes d'exploration, et celui des sciences du passé qui lui fournissent ses objets d'étude.

Ces derniers sont de nature diverse : fossiles animaux et humains, momies naturelles ou artificielles, préparations anatomiques ou pathologiques, séries squelettiques issues de sites archéologiques ou de cimetières contemporains.

Ces pièces ou ces séries ont été constituées en collections, conservées dans différentes structures à vocation scientifique (laboratoires, universités) ou à fonction patrimoniale (services régionaux de l'archéologie, musées) ; ces deux missions sont le plus souvent intriquées, considérant l'interactivité qui doit nécessairement prévaloir entre recherche et patrimoine.

\section{Paléopathologie, paléoépidémiologie et collections squelettiques}

La paléopathologie s'est focalisée à ses débuts sur l'étude de cas remarquables afin de reconnaître des maladies spécifiques sur des restes osseux et d'établir des repères pathologiques temporels et géographiques. Face au développement des grandes collections squelettiques, la paléopathologie s'est alors orientée vers l'analyse des « groupes nosologiques » (grandes catégories de classification des maladies) par des méthodes statistiques utilisées en épidémiologie, afin de définir des fréquences par âge, sexe, chronologie, origine géographique, appartenance culturelle, niveau socio-économique. Cette approche épidémiologique de la paléopathologie, développée par les anthropologues américains dès les années 1930, a été nommée paléoépidémiologie ${ }^{2}$. Cependant, toutes les collections ostéologiques n'ont pas la même valeur pour les recherches paléopathologiques et paléoépidémiologiques, leur intérêt relevant principalement de leur mode de constitution.

Les grandes collections ostéologiques humaines se sont développées en Europe et en Amérique du Nord entre la seconde partie du XIX ${ }^{\mathrm{e}}$ et la première moitié du $\mathrm{XX}^{\mathrm{e}}$ siècle, alimentées par deux grandes spécialités : l'archéologie et l'anatomie. Certaines dépassent plusieurs milliers d'individus et sont conservées dans différentes structures et institutions

Olivier Dutour, directeur d'études à l'EPHE, UMR 5199 PACEA (olivier.dutour@ephe.sorbonne.fr). Hélène Coqueugniot, directeur de recherches au CNRS, directeur d'études cumulant à l'EPHE, UMR 5199 PACEA (helene.coqueugniot@u-bordeaux.fr). 
(universités, musées, muséums d'histoire naturelle). En Amérique du Nord, plusieurs grandes collections archéologiques muséales concernées par la loi fédérale NAGPRA (The Native American Graves Protection and Repatriation Act) ont été ré-inhumées et sont à jamais perdues pour la science.

\section{Les deux grands types de collections d'intérêt paléopathologique}

Les collections ostéologiques humaines actuelles se sont constituées selon deux grandes modalités : anthropologique et pathologique.

\section{Les collections anthropologiques}

Ces collections, qui documentent la variabilité ostéologique humaine contemporaine et passée, proviennent de deux sources principales : «archéologique » et «identifiée ». Elles constituent un objet de recherche méthodologique et appliquée pour les anthropologues, mais également pour les paléopathologistes.

Les collections ostéologiques d'origine archéologique sont composées de restes humains anonymes découverts en contexte archéologique, datant des périodes pré- et protohistoriques jusqu'aux périodes contemporaines. Leur récolement s'est fait lors de fouilles de sauvetage ou de fouilles programmées. En France, ces collections sont le plus souvent conservées dans des dépôts ou « ostéothèques » ayant comme tutelle les services régionaux de l'archéologie, parfois en association avec les structures de recherche (en archéologie et en anthropologie) et les universités. Leur intérêt paléopathologique et paléoépidémiologique varie en fonction de leur conservation, de leur effectif, de la précision de leur chronologie, ainsi que de la durée et du mode de constitution. Les collections provenant d'ensembles funéraires établis sur une large période de temps, à l'intérieur de laquelle les datations archéologiques des sépultures sont peu précises, présentent moins d'intérêt paléoépidémiologique que les collections ayant une résolution centenaire. À l'extrême, les collections anthropologiques résultant d'une accumulation de victimes liées à une mortalité de masse très brève (quelques semaines ou mois) offrent un intérêt paléoépidémiologique tout particulier³.

Les collections ostéologiques identifiées regroupent des sujets décédés généralement entre la fin du XIX ${ }^{\mathrm{e}}$ et le début du $\mathrm{XX}^{\mathrm{e}}$ siècle, associés à des éléments biographiques comme l'état civil, le sexe, l'âge au décès, les causes de la mort, les liens de parenté, la profession, l'anamnèse médicale. Ces collections ont une valeur scientifique bien établie, à la fois pour l'anthropologie biologique ${ }^{4}$ et pour la paléopathologie ${ }^{5}$. Elles constituent un référentiel populationnel moderne pour la variabilité ostéologique chez Homo sapiens, permettant à la fois de modéliser les processus de croissance et de maturation osseuse et dentaire, de tester ou de développer des méthodes de détermination du sexe ou d'estimation de l'âge osseux, de mettre en relation des lésions ou des indicateurs osseux avec des activités ou des pathologies. Leurs effectifs, en général importants (de plusieurs centaines à plusieurs milliers) autorisent des approches statistiques, populationnelles et épidémiologiques.

Ces collections squelettiques identifiées résultent de deux modes de recrutements distincts : un recrutement cimétérial (fin de concession, désaffectation totale ou partielle) et un recrutement anatomique (préparation ostéologique après dissection de corps non réclamés de sujets décédés en milieu hospitalier ou ayant donné leur corps à des laboratoires d'anatomie).

Plusieurs collections ostéologiques d'origine cimétériale existent en Europe, la plus ancienne est celle de Coimbra au Portugal (505 individus), conservée au musée d'Anthropologie de l'Université de Coimbra. La collection Luis Lopes (environ 1700 squelettes) est conservée au Muséum d'histoire naturelle de Lisbonne ; la collection Simon (environ 500 sujets vaudois) fait maintenant partie des collections du laboratoire d'archéologie préhistorique et d'anthropologie de l'Université de Genève. D’autres collections cimétériales de ce type existent en Europe, entre autres à Grenade, Turin, Bologne, Athènes, Budapest et Bucarest. La collection de Spitalfields se distingue par son ancienneté : conservée au Muséum d'histoire naturelle de Londres, elle contient 900 squelettes (le tiers des individus est identifié) de la crypte de Christ Church, avec des décès répartis entre 1646 et 1859 , mais la majorité des individus datent du XVIII ${ }^{\mathrm{e}}$ siècle.

Les principales collections ostéologiques d'origine anatomique sont nord-américaines : la collection Hamann-Todd (Cleveland Museum of Natural History) conserve plus de 3000 squelettes identifiés, la collection Terry (National Museum of Natural History, Smithsonian Institution, Washington, DC), plus de 1 700. En Europe, on peut citer les collections de l'Institut d'anatomie normale (Université de Strasbourg), celles du musée Orfila (transférées à l'Université de Montpellier) et du musée d'Anatomie humaine de l'Université de Turin, particularisées par leurs spécimens ostéologiques juvéniles ${ }^{6}$ (fig. 1 et 2 ).

Ces collections référencées, d'origine cimétériale ou anatomique, ont fait l'objet de très nombreuses études anthropologiques, paléopathologiques et paléoépidémiologiques. Par exemple, la collection de Coimbra a fait l'objet de recherches paléoépidémiologiques portant sur la tuberculose ${ }^{7}$, celle de Cleveland a été utilisée comme référence pour établir des critères pour le diagnostic paléopathologique de plusieurs affections, notamment les cancers osseux ${ }^{8}$. Dans la collection de Spitalfields (crypte), les enfants âgés de plus d'un an présentent presque tous un retard dans la croissance et dans la dentition ${ }^{9}$, évoquant des carences alimentaires au moment du sevrage ; ce qui fait de cette collection un modèle paléoépidémiologique des pathologies carentielles. 


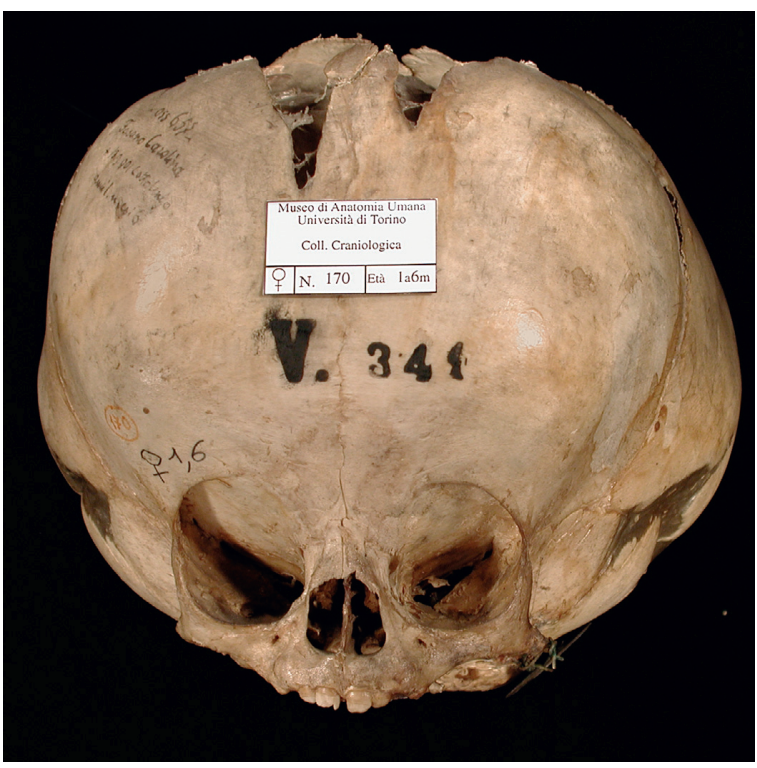

Fig. 1. Crâne d'enfant hydrocéphale (référencé, sujet féminin âgé de 18 mois). Collection du musée d'Anatomie humaine, Université de Turin. (C) H. Coqueugniot.

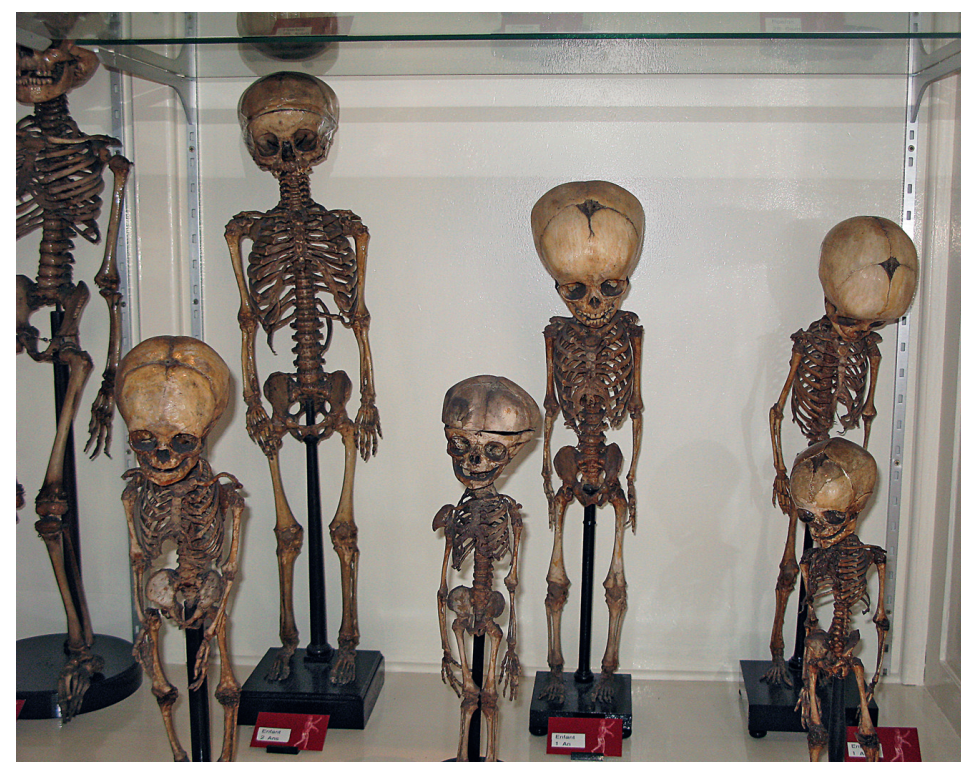

Fig. 2. Squelettes juvéniles montés (d'âge connu). Collections de l'Institut d'anatomie normale de l'Université de Strasbourg. (c) H. Coqueugniot.

\section{Les collections pathologiques}

Les collections à vocation pathologique, références précieuses pour la paléopathologie, sont généralement localisées dans des structures muséales en lien avec l'histoire de la médecine, qu'il s'agisse de laboratoires d'anatomie ou d'anatomo-pathologie, de facultés de médecine, d'universités ou d'institutions hospitalières, mais on peut également les retrouver dans des musées d'histoire naturelle. Elles ont été constituées le plus souvent au sein de laboratoires d'anatomie normale ou pathologique, entre le début du XIX ${ }^{\mathrm{e}}$ siècle et le début du XX ${ }^{\mathrm{e}}$ siècle. Certaines pièces plus rares, notamment certaines reproductions de pièces pathologiques en cires ou en sculptures sur bois, datent de la fin du XVIII ${ }^{\mathrm{e}}$ siècle.

Les restes conservés sont des squelettes entiers, des pièces osseuses, des préparations d'organes, de tissus, de foetus conservés en milieu liquide (bocaux) ou fixés, des reproductions de pièces, d'organes ou d'individus dans des matériaux divers (cires, plâtre, papier mâché verni)...

Chaque pièce est un exemple de pathologie souvent remarquable, fréquemment utilisée en illustration des différents chapitres de manuels de paléopathologie ${ }^{10}$. Ces pièces permettent aux paléopathologistes de visualiser l'histoire naturelle de certaines maladies, à des périodes antérieures aux thérapeutiques modernes, en montrant des expressions pathologiques comparativement plus proches de celles observées sur des restes humains archéologiques que sur les référentiels de pathologie moderne. Ces collections d'anatomie pathologique ont donc principalement un intérêt didactique en paléopathologie, car les pièces conservées sont généralement isolées de leur contexte individuel et populationnel, et de ce fait peu informatives pour l'épidémiologie. Cependant, elles peuvent s'avérer précieuses en paléopathologie génétique, notamment infectieuse, grâce au progrès de la paléogénomique.
On peut entre autres citer en France les collections pathologiques du musée Dupuytren (UPMC-Paris), qui illustrent l'ouvrage méthodologique de Paléopathologie humaine de P. Thillaud, celles de l'Institut d'anatomie pathologique (Université de Strasbourg), documentant de nombreuses pathologies osseuses dans le catalogue de l'exposition «Histoire(s) de squelettes »du Musée de Strasbourg ${ }^{11}$, du musée Ollier des Vans et du musée Testut-Latarjet d'anatomie et d'histoire naturelle médicale (Université de Lyon), du musée Orfila, du musée de l'Homme et, à l'étranger, celles du Muséum d'histoire naturelle de Bâle, du Royal College of Surgeons de Londres, des musées fédéraux d'Anatomie de Vienne et de Göttingen, de l'Armed Force Institute of Pathology de Washington, du Mutter Museum de Philadelphie, du San Diego Museum of Man... la liste n'étant pas exhaustive.

En conclusion, les collections muséales de restes humains, qu'elles soient d'origine archéologique ou anatomique, représentent un matériel d'étude indispensable à la recherche paléopathologique. Cependant, le mode de constitution de ces collections en conditionne fortement l'intérêt : les collections pathologiques illustrent l'histoire naturelle des maladies osseuses et ont une valeur didactique inestimable pour la paléopathologie ; les collections anthropologiques, surtout identifiées, constituent des repères précieux pour les approches méthodologiques en paléoépidémiologie. Les nouvelles méthodes d'imagerie tridimensionnelle contribuent aujourd'hui à valoriser, tout en les préservant, ces collections ostéologiques humaines. 


\section{Notes}

1. Dutour, 2011.

2. Waldron, 1994.

3. Dutour et al., 2007.

4. Coqueugniot, 1999

5. Thillaud, 1996.

6. Coqueugniot, 1999.

7. Santos et Roberts, 2006; Redman et al., 2009.
8. Rothschild et al., 2006.
9. Molleson et al., 1993.
10. Ortner, 2003 ; Thillaud, 1996.
11. Billmann et al., 2005.

\section{Bibliographie}

Coqueugniot H, 1999, Le crâne d'Homo

sapiens en Eurasie : croissance et variation depuis 100000 ans. British

Archaeological Reports S822, Oxford.
Dutour O., 2011, La paléopathologie humaine, éditions du CTHS, Paris.

Dutour O., Maczel M., Ardagna Y., 2007, «Intérêt du modèle peste dans les études paléoépidémiologiques ", M. Signoli, D. Chevé, P. Adalian, G. Boestch, O. Dutour (dir.), La peste : entre épidémies et sociétés, Firenze University Press, Firenze, p. 89-96.

Exp. Strasbourg, 2005: Histoire(s) de squelettes. Archéologie, médecine et anthropologie en Alsace [Exposition Strasbourg, Musée archéologique de Strasbourg, 2005] Schnitzler B., Le Minor J. M., Ludes B., Boes E. (dir.)].

Molleson T. I., Cox M., Waldron A. H., Whittaker D. K., 1993, The Spitalfields Project. Vol. 2. The Anthropology. The Middling Sort. CBA Research Report 86

Ortner D., 2003, Identification of pathological conditions on human skeletal remains, Academic Press, San Diego.

Redman J. E., Shaw M. J., Mallet A. I., Santos A. L., Roberts C. A., Gernaey A. M.,
Minnikin D.E., 2009, "Mycocerosic acid biomarkers for the diagnosis of tuberculosis in the Coimbra Skeletal Collection”, Tuberculosis 89: 267-277.

Rothschild B. M., Hershkovitz I., Dutour O., 1998, "Clues potentially distinguishing lytic lesions of multiple myeloma from those of metastatic carcinoma", American Journal of Physical Anthropology 105 , p. 241-250.

Santos A. L., Roberts C. A., 2006, "Anatomy of a serial killer: differential diagnosis of tuberculosis based on rib lesions of adult individuals from the Coimbra Identified Skeletal Collection, Portugal", American Journal of Physical Anthropology 130, p. 38-49.

Thillaud P., 1996, Paléopathologie humaine, Kronos éditions, Sceaux.

Waldron T., 1994. Counting the Dead. The Epidemiology of Skeletal Populations, Wiley \& Sons, Chichester. 